\title{
A systematic review of diabetes risk assessment tools in sub-Saharan Africa
}

\author{
Edgar Ekure ${ }^{1} \cdot$ Godwin Ovenseri-Ogbomo ${ }^{2,3} \cdot$ Uchechukwu L. Osuagwu $^{2,4}$ (D) $\cdot$ Kingsley E. Agho ${ }^{4,5,10}$. \\ Bernadine N. Ekpenyong ${ }^{6} \cdot K^{2}$ elechi C. Ogbuehi ${ }^{7} \cdot$ Antor O. Ndep ${ }^{8} \cdot$ Patrick Okonji $^{9} \cdot$ Khathutshelo Percy Mashige $^{10}$. \\ Kovin Shunmugan Naidoo ${ }^{10,11}$
}

Received: 15 October 2020 / Accepted: 23 January 2022 / Published online: 12 February 2022

(C) The Author(s) 2022, corrected publication 2022

\begin{abstract}
Objectives To systematically review all current studies on diabetes risk assessment tools used in sub-Saharan Africa (SSA) to diagnose diabetes in symptomatic and asymptomatic patients.

Methods Tools were identified through a systematic search of PubMed, Ovid, Google Scholar, and the Cochrane Library for articles published from January 2010 to January 2020. The search included articles reporting the use of diabetes risk assessment tool to detect individuals with type 2 diabetes in SSA. A standardized protocol was used for data extraction (registry \#177726). Results Of the 825 articles identified, 39 articles met the inclusion criteria, and three articles reported tools used in SSA population but developed for the Western population. None was validated in SSA population. All but three articles were observational studies (136 and 58,657 study participants aged between the ages of 15 and 85 years). The Finnish Medical Association risk tool, World Health Organization (WHO) STEPS instrument, General Practice Physical Activity Questionnaire (GPPAQ), Rapid Eating and Activity Assessment for Patients (REAP), and an anthropometric tool were the most frequently used non-invasive tools in SSA. The accuracy of the tools was measured using sensitivity, specificity, or area under the receiver operating curve. The anthropometric predictor variables identified included age, body mass index, waist circumference, positive family of diabetes, and activity levels.

Conclusions This systematic review demonstrated a paucity of validated diabetes risk assessment tools for SSA. There remains a need for the development and validation of a tool for the rapid identification of diabetes for targeted interventions.
\end{abstract}

Keywords Type 2 diabetes $\cdot$ Validation $\cdot$ Diabetes risk assessment $\cdot$ Africa $\cdot$ Sub-Saharan region $\cdot$ Risk factors

\section{Introduction}

Diabetes mellitus (DM) is a major public health problem globally as the prevalence and burden are uncontrolled in urban

The original online version of this article was revised: The Author's and Editor's corrections were not modified in the original published proof.

Uchechukwu L. Osuagwu

1.osuagwu@westernsydney.edu.au

Edgar Ekure

eekure@gmail.com

Godwin Ovenseri-Ogbomo

godwin.ovenseri-ogbomo@uhi.ac.uk

Kingsley E. Agho

k.agho@westernsydney.edu.au

Bernadine N. Ekpenyong

bekpenyong@unical.edu.ng areas due to significant lifestyle choices [1,2]. Globally, it was reported in 2016 that more than 422 million adults were living with diabetes and estimated to increase by $55 \%$ to over 591.9 million by 2035 [3]. Three-quarters of those with diabetes live

\author{
Kelechi C. Ogbuehi \\ kelechi.ogbuehi@otago.ac.nz \\ Antor O. Ndep \\ drndep@gmail.com \\ Patrick Okonji \\ opatrick@unilag.edu.ng \\ Khathutshelo Percy Mashige \\ mashigek@ukzn.ac.za
}

Extended author information available on the last page of the article 
in low- to middle-income countries (LMICs), and this is projected to increase [4] with a median prevalence estimate at 5\% in sub-Saharan Africa [5].

Due to increasing urbanization, demographics, and nutritional changes in the region [6-8], compounded by a lack of awareness of the lifestyle risk factors for diabetes, inadequate healthcare infrastructure, and lack of access to quality healthcare on the sub-continent $[2,9]$, the prevalence of diabetes is predicted to increase from $4.85 \%$ in 2013 to $5.35 \%$ in 2035 [10]. In addition, the proportion of adults aged 20-79 years with undiagnosed diabetes was estimated to be $90 \%$ of the diabetic population in LMICs compared with $33 \%$ in highincom'e countries [10]. The burden of the disease is evident and keeps increasing $[2,9]$.

Diabetes has become one of four prioritized noncommunicable diseases (NCDs) by the World Health Organization (WHO) [11]. The projected increase in the prevalence of diabetes mellitus (DM) in SSA will exert considerable economic and human resource costs on the healthcare system that is already lacking in funding and trained human resource $[12,13]$. To mitigate this effect, there is a need for an effective, non-invasive screening tool for DM in this region such as the diabetes risk assessment tools which are convenient for early screening and detection of diabetes to avoid diabetes-related morbidity, reduce the cost of healthcare, and improve quality of life [11).

Screening for diabetes in general practice by measuring fasting blood glucose levels is feasible, but expensive, invasive, and time-consuming. This could be more efficient if targeted at high-risk patients [14]. Different strategies have been suggested to improve diabetes detection including opportunistic screening and population-based screening [15].

Over the last decade, many diabetes risk assessment tools used for identifying previously undiagnosed diabetes and individuals at high risk of diabetes have been developed and validated in various countries. However, these tools were developed for different population groups using both community- and population-based studies [16-27], predominantly among Caucasian [20, 28, 29]Biostatistics, Asian [18, 21, 22, 30, 31], and Middle Eastern [32, 33] populations. The tools identified age, sex, obesity, family history of diabetes, and hypertension as the most common factors associated with diabetes [18, 20-22, 28-30, 32, 33]. In a population-based systematic review conducted using 5 qualitative analysis tools (two each from Mexico and Peru and another from Brazil), researchers found that the area under the curve (AUC) ranged from 66 to $72 \%$ and recommended the use of different diabetes risk scores for the Latin American populations [34].

In Africa, there is paucity of data on the use of non-invasive tools for diabetes risk assessment despite the growing population and prevalence of diabetes. For example, in a population-based study conducted in Egypt on 1032 individuals without a history of diabetes, the authors found that a predictive model could easily detect undiagnosed diabetes [35]. Another study conducted on 3094 Mauritian Indians using risk prediction models found that the AUC was $62 \%$ for men and 64\% for women [36]. In Ogun State, Nigeria, using a community-based diabetes risk assessment tool on 56,567 individuals, authors found higher risk scores for diabetes and a higher rate of obesity among females than males. A study by Alebiosu et al. suggested that current evidence should be examined in order to implement diabetes preventative strategies [37]. Overall, these studies [35-37] are in agreement that diabetes assessment tools are limited to the population for which they were developed, and when used in different populations including among SSAs, their validity could be affected resulting in an inferior predictive model.

Therefore, this study was designed to provide evidence on the availability and use of diabetes risk assessment tools in SSA by systematically reviewing all current studies on diabetes risk assessment tools within the region. This was supported by reviewing studies conducted outside SSA countries on diabetes risk assessment tools so that the findings are generalizable to a wider population. Identifying the diabetes risk assessment tool models available in the SSA region would be valuable at the primary care level, for clinicians and public health workers to facilitate early detection of DM among those who are unaware of their status. Also, the study will provide evidence of the risk factors and diabetes risk scores that could be further studied in different SSA countries or integrated into the guidelines for policy-makers as a standard of practice for diabetes screening at the population level. Findings can also be used to develop diabetes prevention and education programs across SSA communities.

\section{Research design and methods}

This study was conducted in conformance with the Preferred Reporting Items for Systematic Reviews and Meta-Analyses (PRISMA) statement on reporting items for systematic reviews and meta-analyses [38] following a protocol for this systematic review which was registered in Prospero (registry \#177726). The search was performed in the following databases: PubMed, Ovid, Google Scholar, and the Cochrane Library. The databases were searched and articles published from January 2000 to January 2020 were included and no language limits were applied. A literature search strategy was developed and implemented using the Population, Intervention, Comparison, Outcome, and Study (PICOS) framework, as shown in Table 1. The search goals were first to identify studies on the development of tools for non-invasive diabetes risk assessment in Africa, and then to expand that search to other continents. Search terms included diabetes risk assessment tools, diabetes mellitus risk assessment 
Table 1 The search strategy for literature selection

\begin{tabular}{ll}
\hline PICOS & Description \\
\hline Population & Indigenous African population aged 15 years and over \\
Intervention & Application of diabetes risk assessment tools \\
Comparison & Comparison of DM risk tools to other previously validated tools \\
Outcome & Accuracy levels of risk assessment tools \\
Study & All studies including but not limited to clinical trials, cohort, \\
& case-control, cross sectional, and reviews \\
\hline
\end{tabular}

tools, diabetes risk assessment tools in Africa, "diabetes" AND "risk assessment tools" AND Africa.

\section{Search strategy and selection criteria}

Two experienced reviewers, EE and GO, independently carried out the searches on two separate dates. PubMed and Ovid databases were searched on January 13, 2020, while Cochrane Library was searched on January 15, 2020. Both reviewers used the same predefined search terms as detailed in the Supplementary file (S1). The search hits were then manually screened for relevance and collated for more detailed scrutiny using predefined inclusion and exclusion criteria. All articles selected by both reviewers were collated for data extraction. A third experienced reviewer, $\mathrm{KO}$, adjudicated disputed articles. The full electronic search strategy for PubMed database, including the limits used, is presented (S1).

\section{Data extraction and quality assessment}

A total of 825 articles were selected for review of the abstracts, and 786 articles were excluded after duplicate removal and after applying the inclusion and exclusion criteria below. Data were extracted after assessing the quality of the studies by using Cochrane collaboration's tool for assessing risk of bias in publications. The following data were extracted from each study where available: authors, year of publication, number of study participants, the location, mean age and/or age range of study participants, sample size, predictor variables (age, body mass index (BMI), waist circumference, waist to height ratio), diabetes risk assessment tools used, accuracy level of the tools and/or risk of developing diabetes, and tool validation.

The article selection process is presented in the PRISMA flow chart (Figure 1). For synthesis of results, qualitative description of data was performed using area under the receiver operating curve (AUC), sensitivity, specificity, positive predictive value, negative predictive value, and odds ratios.

\section{Inclusion and exclusion criteria}

Eligible articles for inclusion were those that reported on the following: individuals 15 years and older; quantitative scores of predictive models; diabetes risk assessment tools developed and used in an African population; validated tools for assessing risk factors for diabetes in an African population; and on specific tools developed for assessing diabetes risk factors in an African population.

Exclusion criteria included articles that reported on validated tools for assessing risk factors for diseases other than DM; on health topics other than DM; and on invasive diabetes risk assessment tools. In addition to those included, articles were also selected and grouped if they included information on diabetes risk assessment tools developed for non-African populations. The same exclusion criteria were adopted but these articles were considered separately from the African studies, only if they met the inclusion criteria.

\section{Results}

\section{Diabetic risk assessment tools in Africa}

Thirty-nine articles met the inclusion criteria for a full review. Only three of those articles were from sub-Saharan Africa [37, $39,40]$ and involved the use of non-invasive diabetes risk assessment tools in local populations (Table 2). The quality of the data in each of the studies conducted in SSA countries is presented in Table 2.

In the current review, the age range of participants in the studies that included Africans was 18-62 years and the sample size ranged from 136 to 58,657 persons. All the studies were observational. The most common non-invasive predictor variables used in the three studies on the African population were age, body mass index (BMI), waist circumference, family history of diabetes, and activity levels. Others include vegetable consumption, waist-to-height ratio (WHtR), and history of use of antihypertensive agents.

\section{Type of tools and tool accuracy}

Five different tools were used in the selected studies including the Finish Medical Association DM risk tool, WHO STEPS instrument, General Practice Physical Activity Questionnaire (GPPAQ), Rapid Eating and Activity Assessment for Patients (REAP), and an anthropometric tool. The accuracy of disease 
Fig. 1 Flow chart of the article selection process. Of the 42 studies that met the inclusion criteria, only three were from subSaharan Africa
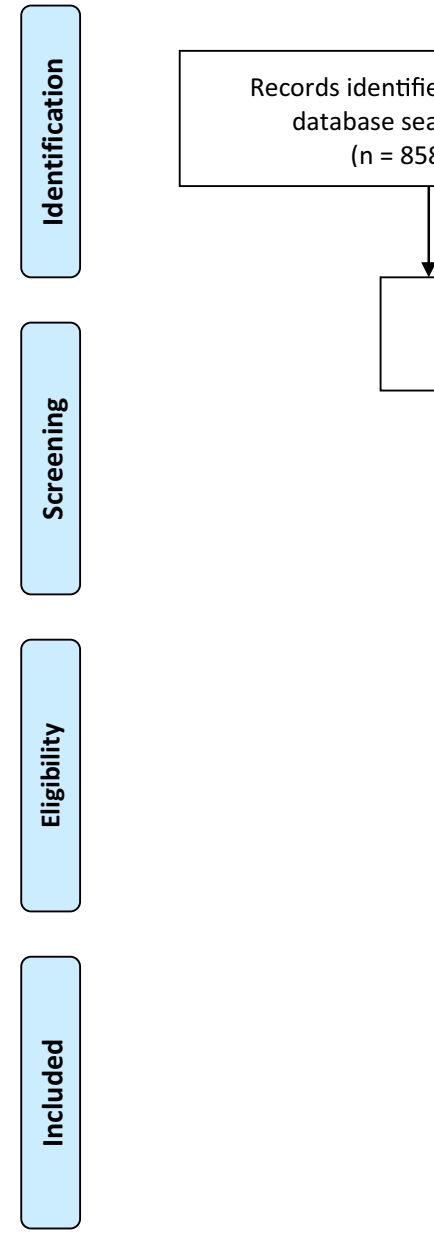

Records after duplicates removed

$(n=825)$

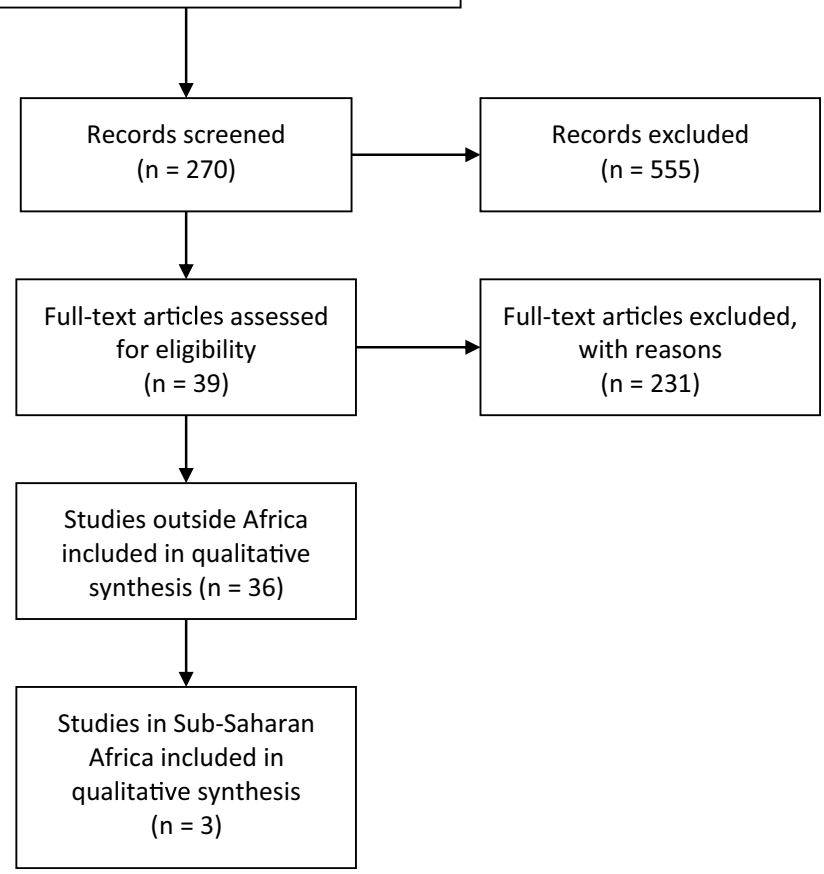

interpretation of the African tools since the tools used in the African studies have been used previously in non-African populations. The continents covered include North America, South America, Europe, Asia, and Australia and two studies from the Middle East Region [54, 55]. The age range of subjects was between 15 and 85 years with a sample size ranging from 44 to 15,768 participants. The most used predictor variables common to all studies were anthropometric variables including BMI, WHtR, and waist circumference (WC), as well as age and family history. Other less commonly used variables were visceral adiposity index (VAI), body adiposity index (BAI), triglyceride (TG) level, smoking, ethnicity, sedentary lifestyle, and hypertension.

\section{Type of tools and accuracy}

Twenty different tools were used in seven studies but the anthropometric and Finnish diabetes risk assessment tools accounted for about $40 \%$ of all tools. Others used locally adapted questionnaires and compared accuracy results with published diabetic risk tools [18, 22, 49, 51, 54, 59, 65]. Most (69.2\%) of the articles reviewed use AUC to report the
Given the few studies in Africa, in addition, we reviewed the 36 articles that met our criteria but were outside of SSA. This was done to broaden the understanding and the depth of 


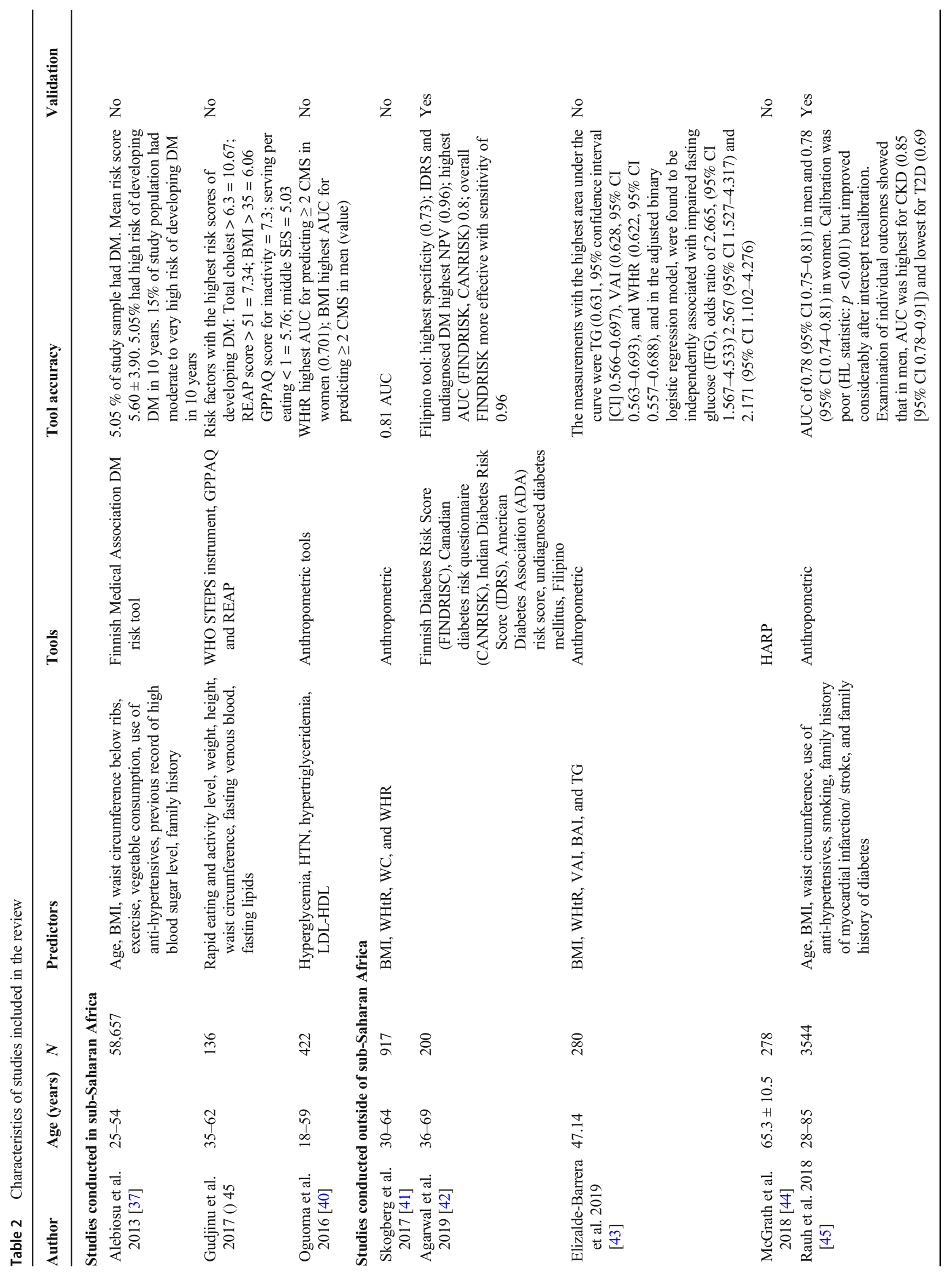



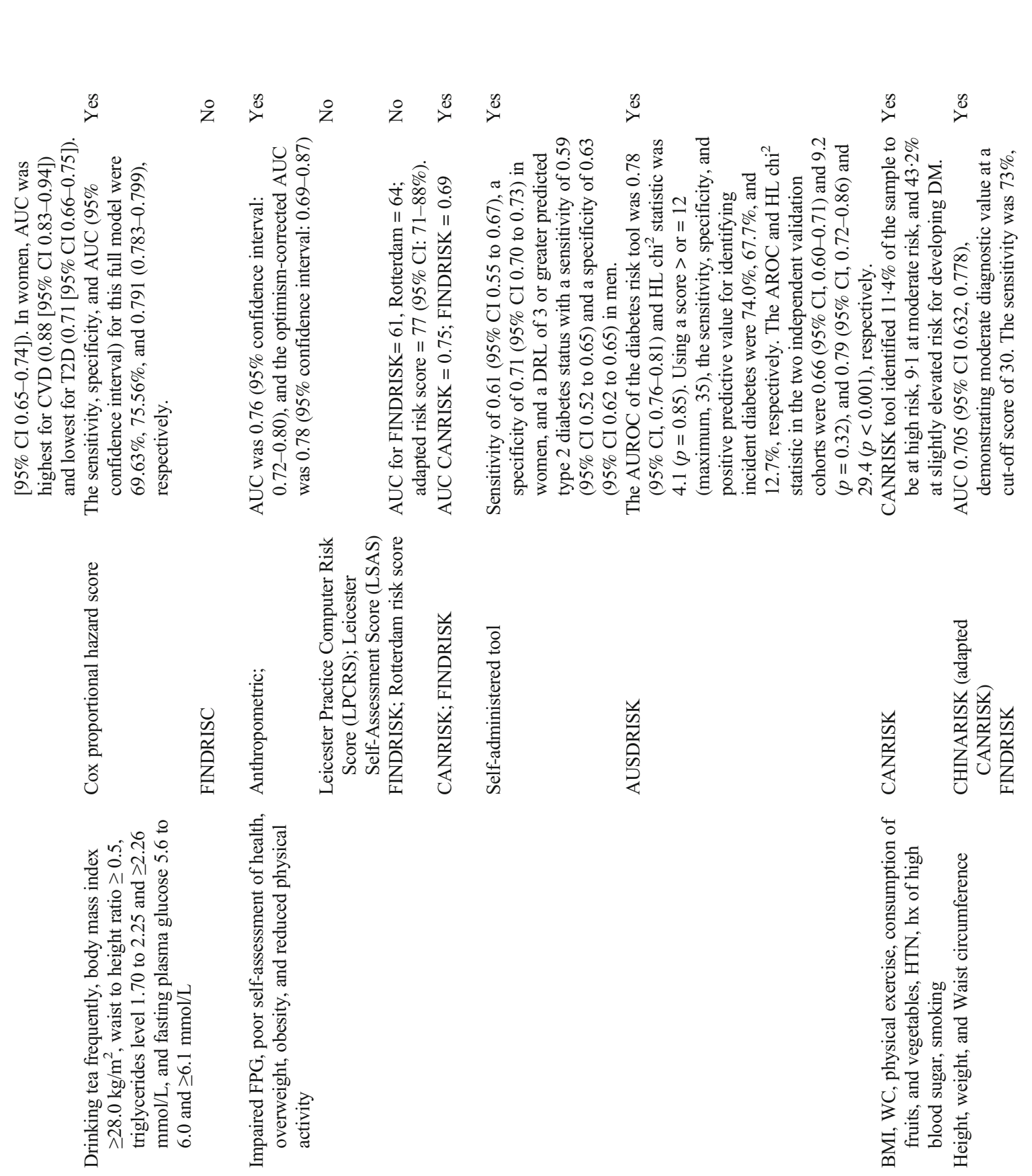

落
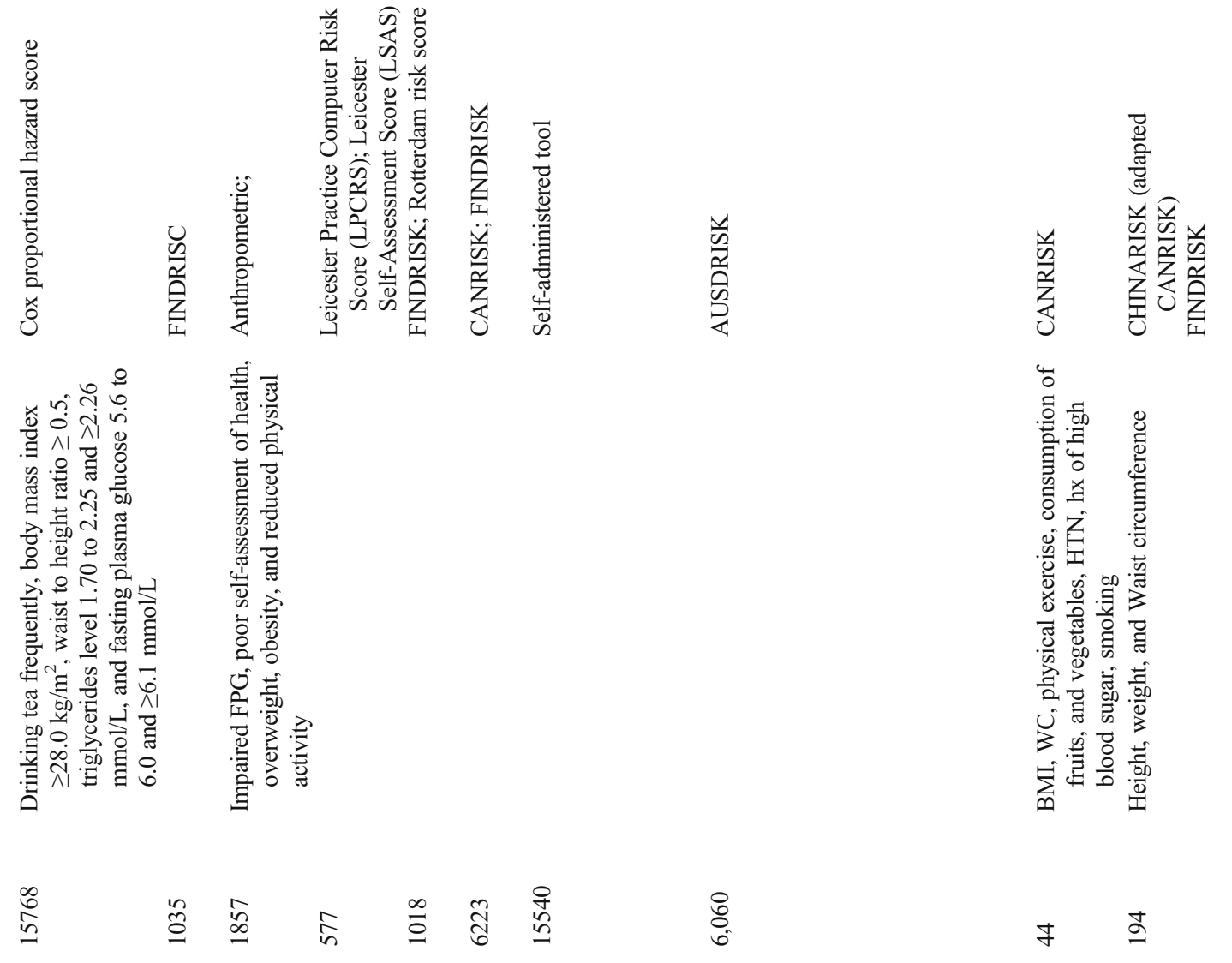

8
$\vdots$
$\substack{V \\ v}$

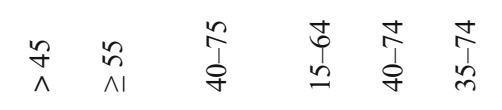

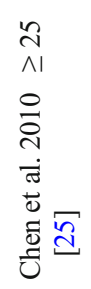

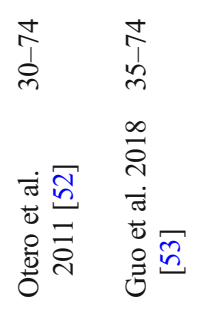




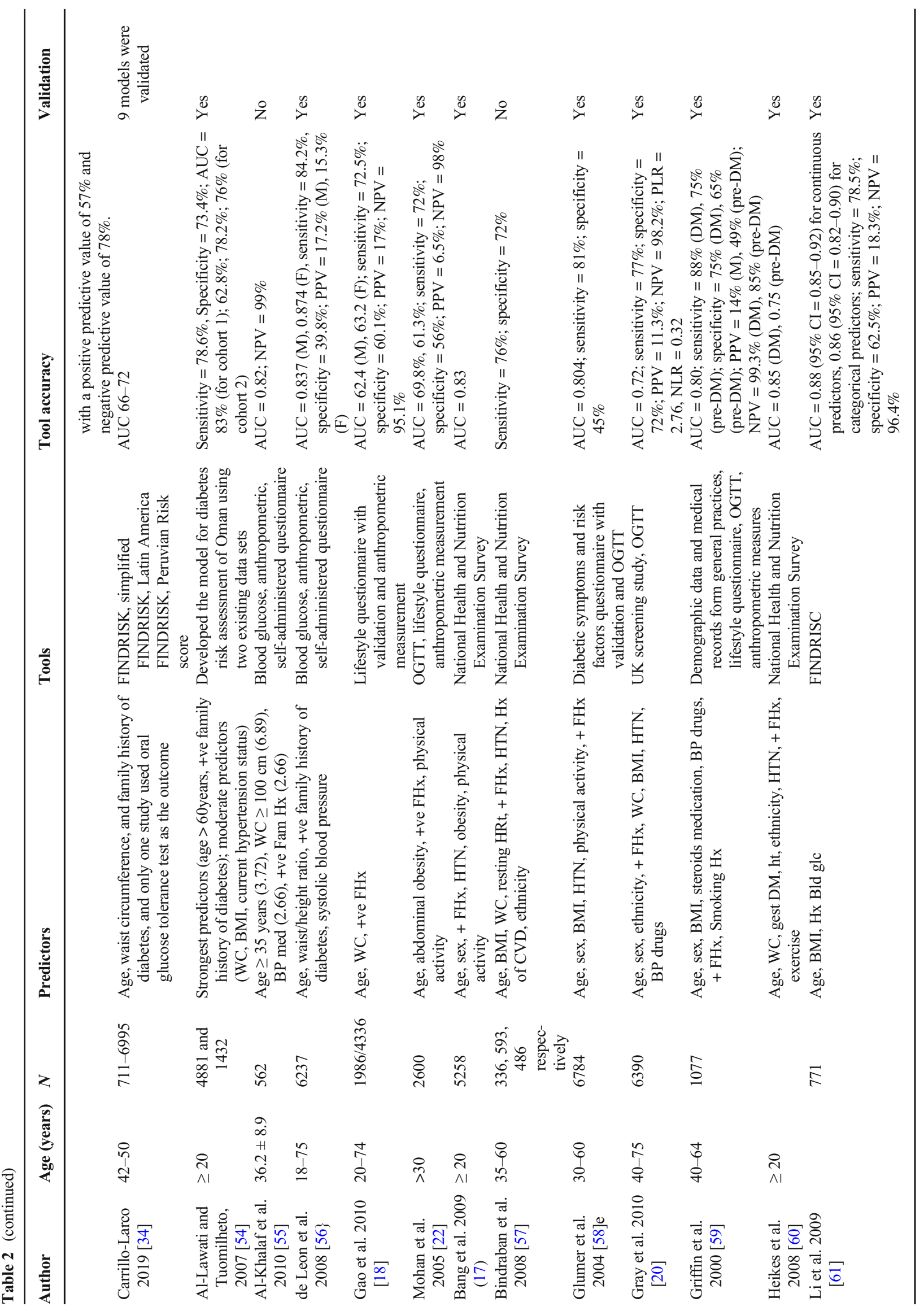




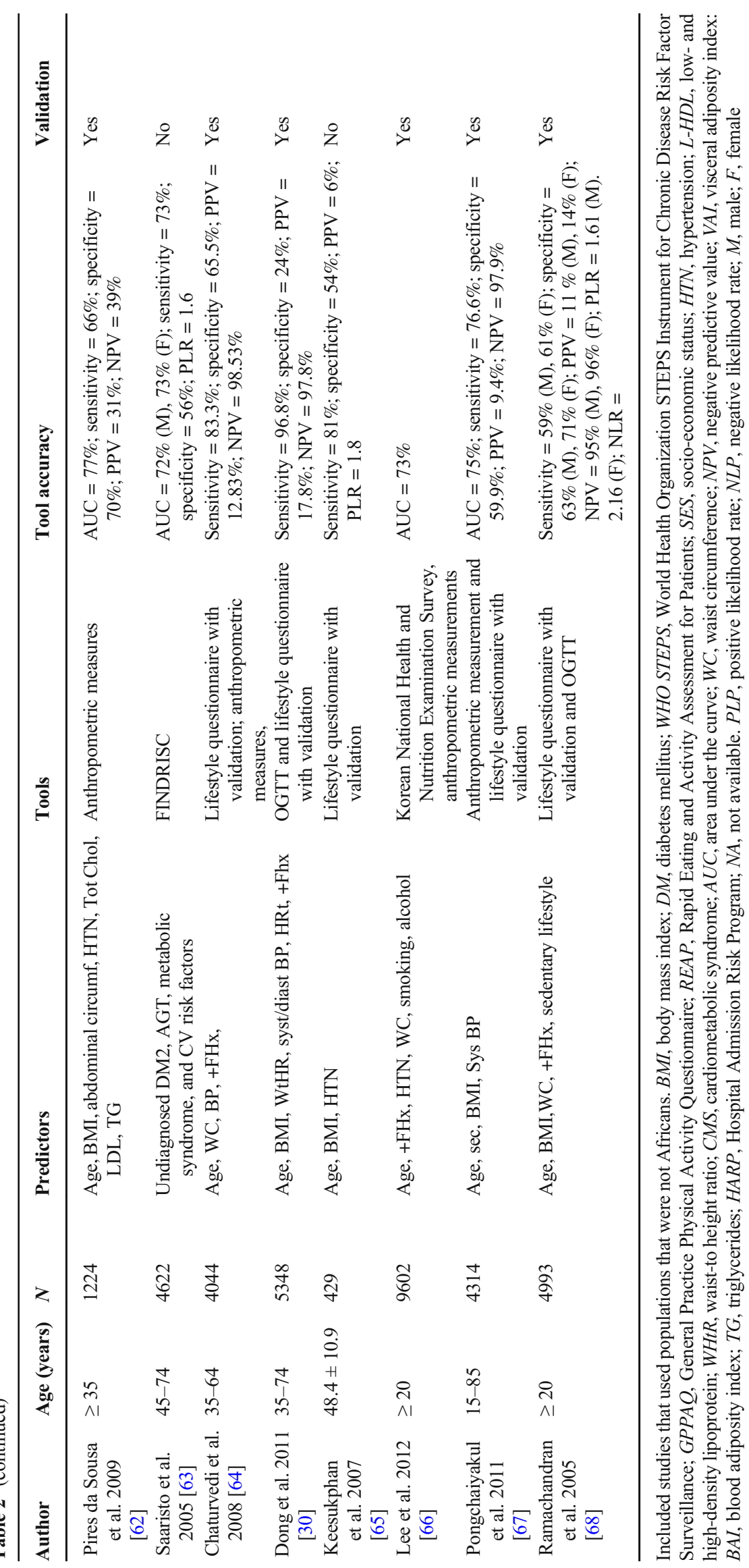


accuracy of the tools with values ranging from 0.60 to 0.88 , while others used sensitivity and specificity for performance validation of the risk assessment tools (51.3\%). Validation of tools was recorded in $77.1 \%$ of the studies using methods such as internal, external, measurement, and split-sample validation.

\section{Discussion}

This study found that the diabetes risk factors pertinent to SSA included waist circumference to height ratio, age, BMI, waist circumference below the ribs, exercise, vegetable consumption, use of anti-hypertensives, previous history of hyperglycemia, positive family history of diabetes, rapid eating, fasting venous blood glucose, and fasting lipids. Different diabetes risk assessment tools have been used in different populations in SSA, but none was developed and/or validated for use in the SSA populations. The findings provide insights and guidelines in a first phase for developing a more population-based diabetes risk assessment tool for SSA based on the reported risk factors in the available studies, and in a second phase for devising and building new risk assessment methods based upon the data collected from the tool design project. The study also reviewed evidence from non-African populations to ensure results are applicable to a wider population. By reviewing both African and non-African studies, this study provides the basis for the selection of the most relevant risk score to be implemented within the population.

It is typical to validate a newly developed tool for use in a population, or adapt a tool previously validated for use in another population. Studies [41] have indicated that available diabetes risk assessment tools perform differently for different populations. This underscores the need to validate a tool prior to its use in a population that is different from its original design. Differences in lifestyle, diet, and other sociodemographic characteristics could account for this variability warranting the need for validation of the tool. In this review, we found that the studies used various validation methods including split-sample cross-validation [50, 69], validation with another external population $[18,45,61,70]$, and bootstrapping [31, 48].

\section{Studies conducted in sub-Saharan Africa}

This review study did not identify a diabetic risk assessment tool specifically designed and validated for the population of SSA and that reported tool accuracy metrics like AUCs, sensitivity and specificity, and positive and negative predictive values. The implication is that clinicians may have to depend on tools developed for other populations to screen for diabetes in the sub-region. Given the vast variation in social, economic, nutritional, demographic, and genetic factors between different populations, and how these factors interplay in the etiology of diabetes, the accuracies of these external diabetic risk assessment tools may be in question. This claim seems to have empirical support from the study done by Skogberg et al. [41] who demonstrated higher accuracy levels among Finns compared to Russian, Somali, and Kudish immigrants when the variables WC and WHtR were used to predict DM. With the prevalence of DM in SSA expected to grow significantly, the need for accurate early detection is imperative. Our study argues strongly in favor of developing such tools.

We found some issues with three studies $[55,59,65]$ identified in this review. Paramount among them was the fact that tools developed for other populations were used in some local SSA populations without validating them for the local populations. The study by Alebiosu et al. [55] provides one such example. They used the Finnish Medical Association diabetes risk assessment (FINDRISK) tool, without validating the tool in the local SSA population. Although they reported a significant correlation between total risk score and fasting blood sugar, they did not report any of the standard measures of tool accuracy like the AUC, sensitivity and specificity, and negative and positive predictive values. Without accurate results, it is not clear if the tool could accurately screen for DM particularly at the early stages in the population they studied.

The WHO STEPS instrument, GPPAQ, and REAP were used in Ghana by another study [51] to assess the risk factors for type $2 \mathrm{DM}$ but did not predict DM directly. Although the tools have utility in associating DM risk factors to scores, robust predictive values were not reported and as with the study by Alebiosu et al. [55], the tools were not validated for the local population. One study [59] reported accuracy levels using the area under the receiver operating characteristics curve (AUC). Anthropometric variables were not used to predict DM directly but of cardiometabolic syndrome (CME), a syndrome which comprises hyperglycemia, hypertension, and hypertriglyceridemia. Although they demonstrated similar AUCs for female and male anthropometric variables like WHtR and BMI, the tool was designed specifically to predict CMS; therefore, its predictive utility for DM remains unclear.

An accurate DM risk assessment tool validated for local SSA populations does not seem to exist yet. However, the few related studies we reviewed showed pertinent anthropometric risk factors for DM that could be used to develop and validate a DM tool in SSA. These anthropometric factors include waist circumference to height ratio, age, BMI, waist circumference below the ribs, exercise, vegetable consumption, use of anti-hypertensives, previous history of hyperglycemia, positive family history of diabetes, and rapid eating. The cut-off values of the anthropometric variables used in the reviewed studies are presupposed normative values for populations outside Africa and could lower the predictive value of the tools in the African population. Perhaps a non-invasive 
tool that uses normative values for WC, WHtR, BMI, dietary intake, exercise regimen, etc., which are pertinent to the local population, could give higher overall accuracy including sensitivity and specificity values. These findings therefore provide insights and guidelines in a first phase for developing a more population-based diabetes risk assessment tool for SSA based on the reported risk factors identified for local populations. A second phase will involve devising and building new risk assessment methods based on the data collected from the tool design project.

\section{Studies conducted outside sub-Saharan Africa}

Regarding the studies conducted outside of SSA, a total of 36 articles on diabetes risk assessment tools were reviewed. In general, the tools were developed in Europe, Asia, the USA, and the Middle East. However, none of the tools was developed or validated for the African population [57]. A similar lack of validated risk assessment tools found in SSA has been noted among some Latin American populations with the study recommending that the health authorities prioritize the development, validation, and implementation of a risk assessment tool [58]. Considering the high prevalence of diabetes and undiagnosed diabetes in the SSA region [10], the health authorities in the various countries should take similar actions by prioritizing the development and validation of a tailored diabetes risk assessment tool. Such tool should ideally be noninvasive, easy to use, free, and easily available to clinicians, public health workers, researchers, and individuals to assess their level of risk for developing diabetes. As with other Western countries, making the tool available on Ministry of Health websites will enable screening and early diagnosis of diabetes to reduce the rate of complications related to undiagnosed diabetes.

The number of cases of diabetes used in the derivation model for risk factors in the studies conducted outside of Africa varied from 48 [60] to 207 [62], and for validation, it was between 29 and 582 cases. Compared to studies in SSA populations, these studies reported similar risk factors outside of Africa [62-64] including age, waist circumference, and family history of diabetes. These were the most common predictors of diabetes and in one study oral glucose tolerance test was the outcome. Of five studies that assessed the use of diabetes risk assessment tools in South America [58], one review study found a high discrimination performance (AUC 70\%, range: 66-72\%) across studies, and the highest metric was always the negative predictive value $[30,60,63$, 64]. Although discrimination estimates in those studies were largely acceptable, calibration metrics were not reported. For countries such as Brazil [66], Mexico [67], and Peru [30] where risk assessment tools were developed and validated both cross-sectionally and prospectively, there was enough scientific evidence to implement these tools as part of the standard of care for type $2 \mathrm{DM}$ screening at the population level [30]. Two studies from the Middle East also reported a high discrimination performance (AUC ranged from 76 to $83 \%$ ) and only one study reported the tool's accuracy in terms of sensitivity and specificity [43] while another study reported the negative predictive value of the tool [41].

Generally, diabetes risk assessment tools cannot be directly transferred from one demographic group to another, due to the variation in factors such as diet and activity levels in different population groups. This is because the accuracy level of the tool varies with racial demographics [61] and since the three studies conducted in the African population used tools that were neither developed nor validated for the SSA population, it may be problematic to assume that their results can be replicated. In addition, only one of the studies reported an accuracy level for the tools used making it impossible to assess the performance of these tools in the SSA population. These findings indicate the need for the development of an accurate and validated non-invasive diabetes risk assessment tool for the SSA population. This tool should be cost-effective and able to identify persons at high risk of developing type 2 diabetes with reasonably high accuracy levels. Such a tool is important because the projected increase in the prevalence of diabetes in Africa will no doubt exert an enormous cost on the healthcare delivery systems in SSA, which are already chronically underfunded and understaffed $[17,18]$.

\section{Limitations and strengths}

One of the limitations of the current review relates to the fact that there was no standardized format for reporting the outcome variables in the different studies. For example, only one of the three studies in sub-Saharan Africa reported the level of accuracy of the screening tool used. The variation in the different tools used in the studies did not allow for comparison of the performance of the screening tools when used for the African population. Another limitation of this study was the small number of published reports on the diabetes risk assessment tools, leading to the inclusion of studies published in nonAfrican regions to enlarge the scope of the discussion as suggested by a reviewer. Despite these limitations, this is the first study to provide evidence on the diabetes risk assessment tools used in SSA. The study reviewed evidence from non-African populations to ensure results are applicable to a wider population. By reviewing both African and non-African studies, this study provides the basis for the selection of the most relevant risk score to be implemented within the population such that the findings can be used as a reference to developing a tool for use among the African population. 


\section{Conclusions}

This comprehensive review of the available literature found that no available diabetes risk assessment tool was developed and validated for the SSA population, despite the disproportionately high prevalence of diabetes in this region and the projected increase. This review found only three articles for the SSA region over a 20 -year period which demonstrates the limited published research on diabetes risk assessment tools in the region. Although the existing European or American diabetes risk tools cannot be adopted in SSA countries without prior validation in the specific population, the findings of this study provided useful evidence of the risk factors and diabetes risk scores that could be further studied in different SSA countries. There is need for practical strategies to address the barriers to the implementation of diabetes risk assessment tools including that a low-cost, reliable screening tool for undiagnosed diabetes be developed and internally validated for the SSA population. The potential for cost and morbidity savings could be significant. Development of such tools should take into account the peculiar demographic characteristics of the sub-region identified in this study. Having a validated diabetes risk assessment tool with sufficiently high sensitivity and specificity will help healthcare policy-makers make informed decisions in the prudent allocation of scarce resources. The tool could then be deployed by trained healthcare workers in the screening of those at risk of diabetes for further clinical examination and possible care and it can be adapted by different SSA countries for validation in different communities. As in many developed countries, such tools can be integrated into the guidelines for policy-makers as a standard of practice for diabetes screening at the population level.

Supplementary Information The online version contains supplementary material available at https://doi.org/10.1007/s13410-022-01045-8.

Author contribution All authors made substantial contributions to the conception or design of the work or the acquisition, analysis, or interpretation of data for the work.

Funding Open Access funding enabled and organized by CAUL and its Member Institutions.

Data availability The data that support the findings of this study are available on request from the corresponding author, ULO.

\section{Declarations}

Ethics approval Not applicable.

Consent to participate Not applicable.

Consent for publication Not applicable.

Competing interests The authors declare no competing interests.
Open Access This article is licensed under a Creative Commons Attribution 4.0 International License, which permits use, sharing, adaptation, distribution and reproduction in any medium or format, as long as you give appropriate credit to the original author(s) and the source, provide a link to the Creative Commons licence, and indicate if changes were made. The images or other third party material in this article are included in the article's Creative Commons licence, unless indicated otherwise in a credit line to the material. If material is not included in the article's Creative Commons licence and your intended use is not permitted by statutory regulation or exceeds the permitted use, you will need to obtain permission directly from the copyright holder. To view a copy of this licence, visit http://creativecommons.org/licenses/by/4.0/.

\section{References}

1. Cho NH, Shaw JE, Karuranga S, Huang Y, da Rocha Fernandes JD, Ohlrogge AW, Malanda B. IDF Diabetes Atlas: global estimates of diabetes prevalence for 2017 and projections for 2045. Diabetes Res Clin Pract. 2018;138:271-81.

2. Mbanya JCN, Motala AA, Sobngwi E, Assah FK, Enoru ST. Diabetes in sub-Saharan Africa. Lancet. 2010;375(9733):2254-66.

3. Guariguata L, Whiting DR, Hambleton I, Beagley J, Linnenkamp U, Shaw JEJDr, et al. Global estimates of diabetes prevalence for 2013 and projections for 2035. 2014;103(2):137-49.

4. Ogurtsova K, da Rocha Fernandes JD, Huang Y, Linnenkamp U, Guariguata L, Cho NH, Cavan D, Shaw JE, Makaroff LE. IDF Diabetes Atlas: Global estimates for the prevalence of diabetes for 2015 and 2040. Diabetes Res Clin Pract. 2017

5. Manne-Goehler J, Atun R, Stokes A, Goehler A, Houinato D, Houehanou C, Hambou MMS, Mbenza BL, Sobngwi E, Balde N, Mwangi JK, Gathecha G, Ngugi PW, Wesseh CS, Damasceno A, Lunet N, Bovet P, Labadarios D, Zuma K, et al. Diabetes diagnosis and care in sub-Saharan Africa: pooled analysis of individual data from 12 countries. Lancet Diabetes \& Endocrinol. 2016;4(11):903-12.

6. Kengne AP, June-Rose McHiza Z, Amoah AG, Mbanya JC. Cardiovascular diseases and diabetes as economic and developmental challenges in Africa. Prog Cardiovasc Dis. 2013;56(3):302-13.

7. Kengne AP, Amoah AG, Mbanya JC. Cardiovascular complications of diabetes mellitus in sub-Saharan Africa. Circulation. 2005;112(23):3592-601.

8. Jakovljevic MB, Milovanovic O. Growing burden of noncommunicable diseases in the emerging health markets: the case of BRICS. Front Public Health. 2015;3:65.

9. Peer N, Kengne A-P, Motala AA, Mbanya JC. Diabetes in the Africa region: an uDiabetic Res Clin Pract. 2014;103(2):197-205.

10. Diabetes I. Brussels: International Diabetes Federation atlas; 2011. International Diabetes Federation. 2012.

11. World Health Organization. Global report on diabetes. 2016. 2017.

12. Mufunda J, Chatora R, Ndambakuwa Y, Nyarango P, Kosia A, Chifamba J, Filipe A, Usman A, Sparks VH. Emerging noncommunicable disease epidemic in Africa: preventive measures from the WHO Regional Office for Africa. Ethn Dis. 2006;16(2):521-6.

13. de Graft AA, Addo J, Ofei F, Bosu WK, Agyemang C. Ghana's burden of chronic non-communicable diseases: future directions in research, practice and policy. Ghana Med J. 2012;46(2):1-3.

14. Lawrence JM, Bennett P, Young A, Robinson AM. Screening for diabetes in general practice: cross sectional population study. BMJ. 2001;323(7312):548-551.

15. Organization $\mathrm{WH}$, editor Screening for type 2 diabetes. Report of the World Health Organization and International Diabetes Federation meeting, 2003; 2003. 
16. Aekplakorn W, Bunnag P, Woodward M, Sritara P, Cheepudomwit S, Yamwong S, Yipintsoi T, Rajatanavin R. A risk score for predicting incident diabetes in the Thai population. Diabetes Care. 2006;29(8):1872-7.

17. Bang H, Edwards AM, Bomback AS, Ballantyne CM, Brillon D, Callahan MA, Teutsch SM, Mushlin AI, Kern LM. Development and validation of a patient self-assessment score for diabetes risk. Ann Intern Med. 2009;151(11):775-83.

18. Gao WG, Dong YH, Pang ZC, Nan HR, Wang SJ, Ren J, et al. A simple Chinese risk score for undiagnosed diabetes. Diabet Med. 2010;27(3):274-281.

19. Glümer C, Carstensen B, Sandbæk A, Lauritzen T, Jørgensen T, Borch-Johnsen K. A Danish diabetes risk score for targeted screening. Diabetes Care. 2004;27(3):727-33.

20. Gray L, Taub N, Khunti K, Gardiner E, Hiles S, Webb D, et al. The Leicester Risk Assessment score for detecting undiagnosed type 2 diabetes and impaired glucose regulation for use in a multiethnic UK setting. Diabet Med. 2010;27(8):887-95.

21. Lee Y-h, Bang H, Kim HC, Kim HM, Park SW, Kim DJ. A simple screening score for diabetes for the Korean population. diabetes care. 2012;35(8):1723.

22. Mohan V, Deepa R, Deepa M, Somannavar S, Datta MA. A simplified Indian Diabetes Risk Score for screening for undiagnosed diabetic subjects. J Assoc Physicians India. 2005;53:759-763.

23. Rahman M, Simmons RK, Harding A-H, Wareham NJ, Griffin SJ. A simple risk score identifies individuals at high risk of developing type 2 diabetes: a prospective cohort study. Fam Pract. 2008;25(3):191-6.

24. Schulze MB, Hoffmann K, Boeing H, Linseisen J, Rohrmann S, Möhlig M, et al. An accurate risk score based on anthropometric, dietary, and lifestyle factors to predict the development of type 2 diabetes. Diabetes Care. 2007;30(3):510-5.

25. Chen L, Magliano DJ, Balkau B, Colagiuri S, Zimmet PZ, Tonkin AM, et al. AUSDRISK: an Australian type 2 diabetes risk assessment tool based on demographic, lifestyle and simple anthropometric measures. Med J Aust. 2010;192(4):197-202.

26. Sathish T, Kannan S, Sarma SP, Thankappan KRJPcd. Peer reviewed: screening performance of diabetes risk scores among Asians and Whites in rural Kerala, India. Prev Chronic Dis. 2013;10. :E37

27. Bernabe-Ortiz A, Smeeth L, Gilman RH, Sanchez-Abanto JR, Checkley W, Miranda JJ, et al. Development and validation of a simple risk score for undiagnosed type 2 diabetes in a resourceconstrained setting. J Diabetes Res. 2016;2016:8790235-.

28. de León AC, Coello SD, Pérez MdCR, Medina MB, González DA, Díaz BB, et al. A simple clinical score for type 2 diabetes mellitus screening in the Canary Islands. Diabet Res Clin Pract. 2008;80(1):128-33.

29. Li J, Bergmann A, Reimann M, Bornstein S, Schwarz PJH, Research M. A more simplified Finnish diabetes risk score for opportunistic screening of undiagnosed type 2 diabetes in a German population with a family history of the metabolic syndrome. Horm Metab Res. 2009;41(02):98-103.

30. Dong J-j, Lou N-j, Zhao J-j, Zhang Z-w, Qiu L-1, Zhou Y, et al. Evaluation of a risk factor scoring model in screening for undiagnosed diabetes in China population. J Zhejiang Univ-Sci B. 2011;12(10):846.

31. Dugee O, Janchiv O, Jousilahti P, Sakhiya A, Palam E, Nuorti JP, Peltonen M. Adapting existing diabetes risk scores for an Asian population: a risk score for detecting undiagnosed diabetes in the Mongolian population. BMC Public Health. 2015;15(1):938.

32. Al Khalaf M, Eid M, Najjar H, Alhajry K, Thalib L. Screening for diabetes in Kuwait and evaluation of risk scores. East Mediterr Health J. 2010. 16(7):725-31.
33. Al-Lawati J, Tuomilehto JJDr, practice c. Diabetes risk score in Oman: a tool to identify prevalent type 2 diabetes among Arabs of the Middle East. 2007;77(3):438-44.

34. Carrillo-Larco RM, Aparcana-Granda DJ, Mejia JR, Barengo NC, Bernabe-Ortiz A. Risk scores for type 2 diabetes mellitus in Latin America: a systematic review of population-based studies. Diab Med. 2019;36(12):1573-84.

35. Tabaei BP, Herman WHJDC. A multivariate logistic regression equation to screen for diabetes: development and validation. Diabetes Care. 2002;25(11):1999-2003.

36. Gao W, Qiao Q, Pitkäniemi J, Wild S, Magliano D, Shaw J, et al. Risk prediction models for the development of diabetes in Mauritian Indians. Diabetes Med. 2009;26(10):996-1002.

37. Alebiosu OC, Familoni OB, Ogunsemi OO, Raimi T, Balogun WO, Odusan $\mathrm{O}$, et al. Community based diabetes risk assessment in Ogun state, Nigeria (World Diabetes Foundation project 08-321). Indian J Endocrinol Metab. 2013;17(4):653.

38. Moher D, Liberati A, Tetzlaff J, Altman DG. Preferred Reporting Items for Systematic Reviews and Meta-Analyses: the PRISMA statement. Int J Surg. 2010;8(5):336-41.

39. Gudjinu HY, Sarfo B. Risk factors for type 2 diabetes mellitus among out-patients in Ho, the Volta regional capital of Ghana: a case-control study. BMC research notes. 2017;10(1):324.

40. Oguoma VM, Nwose EU, Skinner TC, Richards RS, Digban KA, Onyia IC, Anyasodor AE. Anthropometric indices: how they compare in screening of cardiometabolic risks in a Nigerian sub-population. Afr J Med Med Sci. 2016;45(1):91-8.

41. Skogberg N, Laatikainen T, Lilja E, Lundqvist A, Koponen P. Which anthropometric measures best indicate the risk for type 2 diabetes among migrants in Finland? Eur J Pub Health. 2017;27(suppl_3).

42. Agarwal G, Guingona MM, Gaber J, Angeles R, Rao S, Cristobal F. Choosing the most appropriate existing type 2 diabetes risk assessment tool for use in the Philippines: a case-control study with an urban Filipino population. BMC Public Health. 2019;19(1):1169.

43. Elizalde-Barrera CI, Rubio-Guerra AF, Lozano-Nuevo JJ, OlveraGomez JL. Triglycerides and waist to height ratio are more accurate than visceral adiposity and body adiposity index to predict impaired fasting glucose. Diabetes Res Clin Pract. 2019;153:49-54.

44. McGrath RT, Dryden JC, Newlyn N, Pamplona E, O’Dea J, Hocking SL, et al. Utility of the Hospital Admission Risk Programme diabetes risk calculator in identifying patients with type 2 diabetes at risk of unplanned hospital presentations. Intern Med J. 2018;48(10):1198-205.

45. Rauh SP, Rutters F, van der Heijden AA, Luimes T, Alssema M, Heymans MW, et al. External validation of a tool predicting 7-year risk of developing cardiovascular disease, type 2 diabetes or chronic kidney disease. J Gen Intern Med. 2018;33(2):182-8.

46. Zhang H, Wang C, Ren Y, Wang B, Yang X, Zhao Y, et al. A riskscore model for predicting risk of type 2 diabetes mellitus in a rural Chinese adult population: a cohort study with a 6-year follow-up. Diabetes Metab Res Rev. 2017;33(7).

47. Bould K, Scott S, Dunne S, Asimakopoulou K. Uptake of screening for type 2 diabetes risk in general dental practice; an exploratory study. Br Dent J. 2017;222(4):293-6.

48. Liu PJ, Ma F, Lou HP, Chen Y. Visceral adiposity index is associated with pre-diabetes and type 2 diabetes mellitus in Chinese adults aged 20-50. Ann Nutr Metab. 2016;68(4):235-43.

49. Khunti K, Gillies CL, Dallosso H, Brady EM, Gray LJ, Kilgallen G, Willis A, Zafar A, Davies MJ. Assessment of response rates and yields for two opportunistic tools for early detection of non-diabetic hyperglycaemia and diabetes (ATTEND). A randomised controlled trial and cost-effectiveness analysis. Diabetes Res Clin Pract. 2016;118:12-20.

50. Robinson C, Agarwal G, Nerenberg K. Validating the CANRISK prognostic model for assessing diabetes risk in Canada's multi- 
ethnic population. Chronic diseases and injuries in Canada. 2011;32(1).

51. Xie J, Hu D, Yu D, Chen C-S, He J, Gu D. A quick self-assessment tool to identify individuals at high risk of type 2 diabetes in the Chinese general population. J Epidemiol Community Health. 2010;64(3):236-42.

52. Otero LM, Fong M, Papineau D, Thorne S, Zanetti ML. Testing a prediabetes screening approach for a Latin American population in Vancouver, Canada. J Nurs Healthc Chronic Illn. 2011;3(3):329-38.

53. Guo J, Shi Z, Chen JL, Dixon JK, Wiley J, Parry M. Translation and validation of the Canadian diabetes risk assessment questionnaire in China. Public Health Nurs. 2018;35(1):18-28.

54. Al-Lawati JA, Tuomilehto J. Diabetes risk score in Oman: a tool to identify prevalent type 2 diabetes among Arabs of the Middle East. Diabetes Res Clin Pract. 2007;77(3):438-44.

55. Al Khalaf MM, Eid MM, Najjar HA, Alhajry KM, Doi SA, Thalib L. Screening for diabetes in Kuwait and evaluation of risk scores. East Mediterr Health J. 2010;16(7):725-31.

56. Cabrera de León A, Coello SD, Rodríguez Pérez Mdel C, Medina MB, Almeida González D, Díaz BB, et al. A simple clinical score for type 2 diabetes mellitus screening in the Canary Islands. Diabetes Res Clin Pract. 2008;80(1):128-33.

57. Bindraban NR, van Valkengoed IGM, Mairuhu G, Holleman F, Hoekstra JBL, Michels BPJ, et al. Prevalence of diabetes mellitus and the performance of a risk score among Hindustani Surinamese, African Surinamese and ethnic Dutch: a cross-sectional populationbased study. BMC Public Health. 2008;8:271-.

58. Glümer C, Carstensen B, Sandbaek A, Lauritzen T, Jørgensen T, Borch-Johnsen K. A Danish diabetes risk score for targeted screening: the Inter99 study. Diabetes Care. 2004;27(3):727-33.

59. Griffin SJ, Little PS, Hales CN, Kinmonth AL, Wareham NJ. Diabetes risk score: towards earlier detection of type 2 diabetes in general practice. Diabetes Metab Res Rev. 2000;16(3):164-71.

60. Heikes KE, Eddy DM, Arondekar B, Schlessinger L. Diabetes risk calculator: a simple tool for detecting undiagnosed diabetes and prediabetes. Diabetes Care. 2008;31(5):1040-5.

61. Li J, Bergmann A, Reimann M, Bornstein SR, Schwarz PE. A more simplified Finnish diabetes risk score for opportunistic screening of undiagnosed type 2 diabetes in a German population with a family history of the metabolic syndrome. Horm Metab Res. 2009;41(2):98-103.

62. Pires de Sousa AG, Pereira AC, Marquezine GF, Marques do Nascimento-Neto R, Freitas SN, de CNRL, et al. Derivation and external validation of a simple prediction model for the diagnosis of type 2 diabetes mellitus in the Brazilian urban population. Eur $\mathrm{J}$ Epidemiol. 2009;24(2):101-9.

63. Saaristo T, Peltonen M, Lindström J, Saarikoski L, Sundvall J, Eriksson JG, Tuomilehto J. Cross-sectional evaluation of the Finnish Diabetes Risk Score: a tool to identify undetected type 2 diabetes, abnormal glucose tolerance and metabolic syndrome. Diab Vasc Dis Res. 2005;2(2):67-72.

64. Chaturvedi V, Reddy KS, Prabhakaran D, Jeemon P, Ramakrishnan L, Shah P, et al. Development of a clinical risk score in predicting undiagnosed diabetes in urban Asian Indian adults: a population-based study. CVD Prevention and Control. 2008;3(3):141-51.

65. Keesukphan P, Chanprasertyothin S, Ongphiphadhanakul B, Puavilai $\mathrm{G}$. The development and validation of a diabetes risk score for high-risk Thai adults. J Med Assoc Thail. 2007;90(1):149-54.

66. Lee YH, Bang H, Kim HC, Kim HM, Park SW, Kim DJ. A simple screening score for diabetes for the Korean population: development, validation, and comparison with other scores. Diabetes Care. 2012;35(8):1723-30.

67. Pongchaiyakul C, Kotruchin P, Wanothayaroj E, Nguyen TV. An innovative prognostic model for predicting diabetes risk in the Thai population. Diabetes Res Clin Pract. 2011;94(2):193-8.

68. Ramachandran A, Snehalatha C, Vijay V, Wareham NJ, Colagiuri S. Derivation and validation of diabetes risk score for urban Asian Indians. Diabetes Res Clin Pract. 2005;70(1):63-70.

69. Xie J, Hu D, Yu D, Chen C-S, He J, Gu D. A quick self-assessment tool to identify individuals at high risk of type 2 diabetes in the Chinese general population. J Epidemiol Community Health. 2010;64(3):236-42.

70. Ruige JB, de Neeling JN, Kostense PJ, Bouter LM, Heine RJ. Performance of an NIDDM screening questionnaire based on symptoms and risk factors. Diabetes Care. 1997;20(4):491-6.

Publisher's note Springer Nature remains neutral with regard to jurisdictional claims in published maps and institutional affiliations. 


\section{Affiliations}

\section{Edgar Ekure ${ }^{1} \cdot$ Godwin Ovenseri-Ogbomo ${ }^{2,3} \cdot$ Uchechukwu L. Osuagwu ${ }^{2,4}$ (D) Kingsley E. Agho 4,5,10 • Bernadine N. Ekpenyong ${ }^{6} \cdot$ Kelechi C. Ogbuehi $^{7}$ - Antor O. Ndep $^{8} \cdot$ Patrick Okonji $^{9} \cdot$ Khathutshelo Percy Mashige $^{10}$. Kovin Shunmugan Naidoo ${ }^{10,11}$}

Edgar Ekure

eekure@gmail.com

Godwin Ovenseri-Ogbomo

godwin.ovenseri-ogbomo@uhi.ac.uk

Kingsley E. Agho

k.agho@westernsydney.edu.au

Bernadine N. Ekpenyong

bekpenyong@unical.edu.ng

Kelechi C. Ogbuehi

kelechi.ogbuehi@otago.ac.nz

Antor O. Ndep

drndep@gmail.com

Patrick Okonji

opatrick@unilag.edu.ng

Khathutshelo Percy Mashige

mashigek@ukzn.ac.za

1 Department of Biomedicine, Salus University, 8360 Old York Road, Elkins Park, PA 19027, USA

2 Division African Vision Research Institute, African Vision Research Institute, University of KwaZulu-Natal, Durban, South Africa

3 Department of Optometry, Centre for Health Sciences, University of the Highlands and Islands, Old Perth Road, Inverness IV2 3JH, United Kingdom

4 Translational Health Research Initiative (THRI), School of Medicine, Western Sydney University, Campbelltown, NSW 2560, Australia

5 School of Health Sciences, Western Sydney University, Campbelltown, NSW 2560, Australia
6 Epidemiology and Biostatistics Unit, Department of Public Health, University of Calabar, Calabar, Nigeria

7 Department of Medicine, Dunedin School of Medicine, University of Otago, Dunedin, New Zealand

8 Department of Public Health, Faculty of Allied Medical Sciences, College of Medical Sciences, University of Calabar, Calabar, Cross River State, Nigeria

9 Research and Innovation Office, University of Lagos, Akoka, Nigeria

10 African Vision Research Institute, University of KwaZulu-Natal Durban, Durban, South Africa

11 School of Optometry and Vision Science, University of New South Wales, Sydney, New South Wales, Australia 